\title{
A NONSOLVABLE GROUP OF EXPONENT 5
}

BY SEYMOUR BACHMUTH, ${ }^{1}$ HORACE Y. MOCHIZUKI, ${ }^{1}$ AND DAVID WALKUP

Communicated by Paul F. Bateman, November 17, 1969

THEOREM 1. There exists a group $\mathcal{G}$ of exponent 5 which is locally nilpotent, but not nilpotent. In particular, $\mathrm{g}$ is not solvable.

Thus there exist varieties which are nonsolvable, but locally finite and locally solvable.

To prove Theorem 1, we first show that a certain ring is not nilpotent. Let $R$ be the free associative ring of characteristic 5 generated by noncommuting indeterminates $x_{1}, x_{2}, \cdots$, and let $L$ be the Lie ring in $R$ generated by $x_{1}, x_{2}, \ldots$ where addition in $L$ is the same as in $R$ and Lie multiplication is commutation $[x, y]=x y-y x$ in $R$. An element of $L$ will be called a Lie element.

THEOREM 2. If we impose on $R$ the following identical relations for Lie elements $x$ and $y$ :

$$
x^{3}=0
$$

and

$$
x^{2} y-3 x y x+3 y x^{2}=0
$$

then the resulting ring is not nilpotent.

REMARK. Higgins in [3] showed that (i) and (ii) holds in the endomorphism ring of the additive group of a Lie ring satisfying the third Engel condition.

Also worth mentioning is the following result which is equivalent to Theorem 2 as shown in Walkup [8].

THEOREM 3. There exists a Lie ring of characteristic 5 which satisfies the third Engel condition and which is not nilpotent.

G. Higman [4] and A. I. Kostrikin [5] showed that a Lie ring of characteristic 5 satisfying the fourth Engel condition is locally nilpotent, and in view of Theorem 3, this is the best one can say.

AMS Subject Classifications. Primary 1632, 2027, 2040; Secondary 1730, 2008.

Key Words and Phrases. Lie rings, Engel condition, Burnside problem, nilpotency index of rings, nonsolvable locally solvable varieties, exponent 5 .

1 The work of the first two authors was supported in part by National Science Foundation Grant GP-11771. 
Kostrikin [6], in fact, was able to prove the very general theorem that a Lie ring satisfying the $n$th Engel condition and having prime characteristic $p>n$ is locally nilpotent.

In getting away from the finite generation condition, P. Higgins [3] and Heineken [2], showed that an associative ring with characteristic prime to $2,3,5$ and 7 in which the cube of every Lie element is zero must be nilpotent of index at most $3^{9}$. D. Walkup [8] in his thesis improved this result in two ways. First he showed that no restriction on the prime 7 is necessary and secondly that the nilpotency index can be greatly reduced. Specifically he showed

THEOREM 4. Let $R^{\prime}$ be the free associative ring generated by noncommuting indeterminates $x_{1}, x_{2}, \cdots$, with coefficients in a ring in which division by 2, 3, and 5 are possible and the cubes of all Lie elements are zero. Then $R^{\prime}$ is nilpotent of index at most 9.

We sketch the proof of Theorem 2 and the deduction of Theorem 1 from it.

Relations (i) and (ii) together are equivalent to the "Higgins relations",

$$
x y z+x z y=y z x+z y x=2(y x z+z x y)
$$

for all (homogeneous) Lie elements $x, y, z$.

Let $H$ be the ideal of $R$ generated by (iii). Denote by $R_{n}$ the vector subspace of $R$ with basis consisting of all monomials of total degree $2 n$ and degree 2 in each indeterminate $x_{i}, i=1,2, \cdots, n$. Making use of the relations (iii), we are able to establish inductively for each $n \geqq 2$ the existence of a linear transformation $\alpha$ of $R_{n}$ onto $Z_{5}$, the integers modulo five, which satisfies:

(a) $\alpha\left(x_{1}^{2} x_{2}^{2} \cdots x_{n}^{2}\right)=1$.

(b) $\alpha(M N)=\alpha(N M)$, where $M$ and $N$ are any monomials such that $M N$ is in $R_{n}$.

(c) $\alpha(M)=\alpha\left(M^{\prime}\right)$, where $M$ is any monomial in $R_{n}$ and $M^{\prime}$ is the monomial obtained from it by permuting (the names of) the indeterminates.

(d) $\alpha(M)=\alpha\left(M^{T}\right)$, where $M$ is any monomial in $R_{n}$ and $M^{T}$ is the monomial obtained from it by reversing the order of the factors (of degree 1).

(e) $\alpha[M(x y z+x z y)]=\alpha[M(y z x+z y x)]=2 \alpha[M(y x z+z x y)]$, where $x, y$, and $z$ are chosen from among the generators $x_{i}$ and $M$ is any monomial such that the indicated products are in $R_{n}$.

We then show that the kernel of $\alpha$, say $S_{n}$, contains $H \cap R_{n}$, i.e., (e) holds for all Lie elements $x, y$ and $z$ such that $M x y z$ is in $R_{n}$. 
Since $x_{1}^{2} x_{2}^{2} \cdots x_{n}^{2}$ is not in $S_{n}$ and hence not in $H, R$ is not nilpotent modulo $H$, establishing Theorem 2 .

Using Bruck's notation in $\S 3$ of [1], we can show that Theorem 2 implies that $R$ is not nilpotent modulo the permutation ideal of $T_{k}$. Then, by Theorem 4.3 of [1] this last fact implies the negation of the statement $R(3, \pi)$ in Bruck's notes [1], i.e.,

THEOREM 5. There exists a group ring $Z_{5} G$ over the field $Z_{5}$ of integers modulo 5 such that the augmentation ideal of $Z_{5} G$ is not nilpotent modulo the ideal I generated by all elements $(g-1)^{3}$ with $g$ in $G$.

To complete the proof of Theorem 1, we use a standard construction. Let $Z_{5} G$ and $I$ be as in Theorem 5. Define group $g$ to be the set of all ordered pairs $\{g, r\}, g \in G, r \in Z_{5} G / I$ with the multiplication

$$
\{g, r\}\{h, s\}=\{g h, r h+s\} .
$$

An easy check shows that $\mathcal{G}$ has exponent 5. If $a=\{1,1\}$ and $b_{i}=\left\{g_{i}, 0\right\}$, then the commutator

$$
\left(a, b_{1}, b_{2}, \cdots, b_{n}\right)=\left\{1,\left(g_{1}-1\right)\left(g_{2}-1\right) \cdots\left(g_{n}-1\right)\right\} .
$$

Since the augmentation ideal of $Z_{5} G$ is not nilpotent modulo $I, g$ is not nilpotent, and hence by a theorem of Tobin [7], $९$ is not solvable. Thus, $G$ is also nonsolvable.

\section{REFERENCES}

1. R. H. Bruck, Engel conditions in groups and related questions, Lecture notes, Australian National University, Canberra, 1963.

2. H. Heineken, Endomorphismenringe und engelsche Elemente, Arch. Math. 13 (1962), 29-37. MR 26 \#206.

3. P. J. Higgins, Lie rings satisfying the Engel condition, Proc. Cambridge Philos. Soc. 50 (1954), 8-15. MR 15, 596.

4. G. Higman, On finite groups of exponent five, Proc. Cambridge Philos. Soc. 52 (1956), 381-390. MR 18, 377.

5. A. I. Kostrikin, Solution of a weakened problem of Burnside for exponent five, Izv. Akad. Nauk SSSR Ser. Mat. 19 (1955), 233-244. (Russian) MR 17, 126; MR 17, 1436.

6. —, The Burnside problem, Izv. Akad. Nauk SSSR Ser. Mat. 23 (1959), 3-34; English transl., Amer. Math. Soc. Transl. (2) 36 (1964), 63-99. MR 24 \#A1947.

7. S. Tobin, On a theorem of Baer and Higman, Canad. J. Math. 8 (1956), 263-270. MR 17, 1182.

8. D. W. Walkup, Lie rings satisfying Engel conditions, Thesis, University of Wisconsin, Madison, Wis., 1963, University Microfilms, Ann Arbor, Michigan.

University of California, Santa Barbara, California 93106 and Boeing Research Laboratories, Seattle, Washington 98124 